\title{
MICROWAVE-ASSISTED SYNTHESIS OF TETRAZOLE BASED BIPHENYLS DERIVATIVES AND THEIR ANTIMICROBIAL ACTIVITY
}

\author{
D. Ashok ${ }^{1, *}$, Nalaparaju Nagaraju ${ }^{1}$, M. Ram Reddy ${ }^{1}$ Ravinder $^{1}$ Dharavath, \\ K. Ramakrishna ${ }^{1}$ and M. Sarasija ${ }^{2}$ \\ ${ }^{1}$ Green and Medicinal Chemistry Laboratory, Department of chemistry, Osmania University, \\ Hyderabad-500007, (Telangana) India. \\ ${ }^{2}$ Department of Chemistry, Satavahana University, Karimnagar-505001, (Telangana) India. \\ *E-mail: ashokdou@gmail.com
}

\begin{abstract}
An environmentally friendly method has been developed for substituted (E)-(3-(4-(1H-tetrazole-5$y l$ styryl)benzofuran-2-yl)([1,1'-biphenyl]-4-yl)methanone scaffolds by Suzuki cross-coupling reaction in an aqueous medium under both microwave irradiation and conventional heating methods. The structures of the synthesized scaffolds were characterized by IR, NMR, and mass spectral analysis. Furthermore, the synthesized scaffolds were screened for their in-vitro antibacterial, antifungal activities and most of the scaffolds exhibited better activity compared to standard drugs.
\end{abstract}

Keywords: Aromatic Boronic Acid, Suzuki Cross-coupling Reaction, Microwave Irradiation, Antimicrobial Activity

(C) RASĀYAN. All rights reserved

\section{INTRODUCTION}

Tetrazole is a key structural motif in heterocyclic chemistry, it has been found in many drug molecules ${ }^{1-2}$ and these possess wide a range of biological activities. ${ }^{3-10}$ Aryl-aryl bond development is very significant in organic synthesis and has a broad range of applications in industrial interest, as well as the synthesis of pharmaceuticals, polymers, herbicides and other material. ${ }^{11}$ The palladium-catalyzed by aryl halides with aryl boronic acid known as Suzuki cross-coupling reaction, is one of the most important and potent methods for the construction of biphenyl and polyaryls, ${ }^{12-14}$ due to the broad functional group tolerance and the low toxic reagents and the probability of using of green solvents as reaction medium.

The current years, organic synthesis is carried out in aqueous phase or containing water or mixture of organic/water as the solvent has been traditional in the field of organic chemistry. One of the most significant techniques is microwave irradiation ${ }^{16,17}$ which useful in organic synthesis and has been the eco-friendly, inflammable, enhanced rate of reaction, reduced side products, improved product yield as compared to conventional heating. ${ }^{18}$ Consequently, the microwave-assisted reactions using green solvents like water, to easily achieve the aim of green chemistry.

Encourage of biological activity and microwave irradiation techniques; we decided the synthesis of novel tetrazole containing benzofuran scaffolds $5 \mathbf{a}-\mathbf{5} \mathbf{h}$ and respective aryls $7 \mathbf{a}-7 \mathbf{j}$ carried out under both conventional heating, microwave irradiation methods and evaluated their antimicrobial activity.

\section{Materials and Methods}

\section{EXPERIMENTAL}

Melting points were determined in an open glass capillary tube on a Gallen-Kamp MFB-595 apparatus and are uncorrected. The IR spectra were taken on a Perkin-Elmer FT-IR-8400s, using samples in $\mathrm{KBr}$

Rasayan J. Chem., 13(1), 601-609(2020)

http://dx.doi.org/10.31788/RJC.2020.1315490

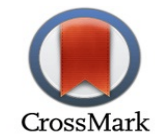


RASĀYAN J. Chem.

Vol. 13 | No. 1 |601 - 609| January - March | 2020

disks. Microwave reactions were carried out in the milestone multi SYNTH microwave system. The ${ }^{1} \mathrm{H}$ NMR (400 MHz) and ${ }^{13} \mathrm{C}$ NMR (100 MHz) spectra were recorded on Bruker Avance II 400 spectrometer. Mass spectra were recorded on SHIMADZU LCMS 2020 mass spectrometer.

\section{RESULTS AND DISCUSSION}

The compounds 3a-3g on reaction with different substituted phenacyl bromides in the presence of anhydrous potassium carbonate as a base in dry acetone as a solvent and refluxed to obtained new compounds 5a-5h. Latter compound $\mathbf{5 h}$ on treated with substituted aryl boronic acids $\mathbf{6 a - 6} \mathbf{j}$ in presence of $\mathrm{Pd}\left(\mathrm{PPh}_{3}\right)_{4}$ as catalyst and $\mathrm{Na}_{2} \mathrm{CO}_{3}$ as a base to obtain the target molecules 7a-7j represented in Scheme-1. All Synthesised scaffolds $\mathbf{5 a - 5 h}$ and $\mathbf{7 a - 7} \mathbf{j}$ produced better yield along with shorter reaction duration using microwave irradiation method as compared to the conventional heating method (Table-1).
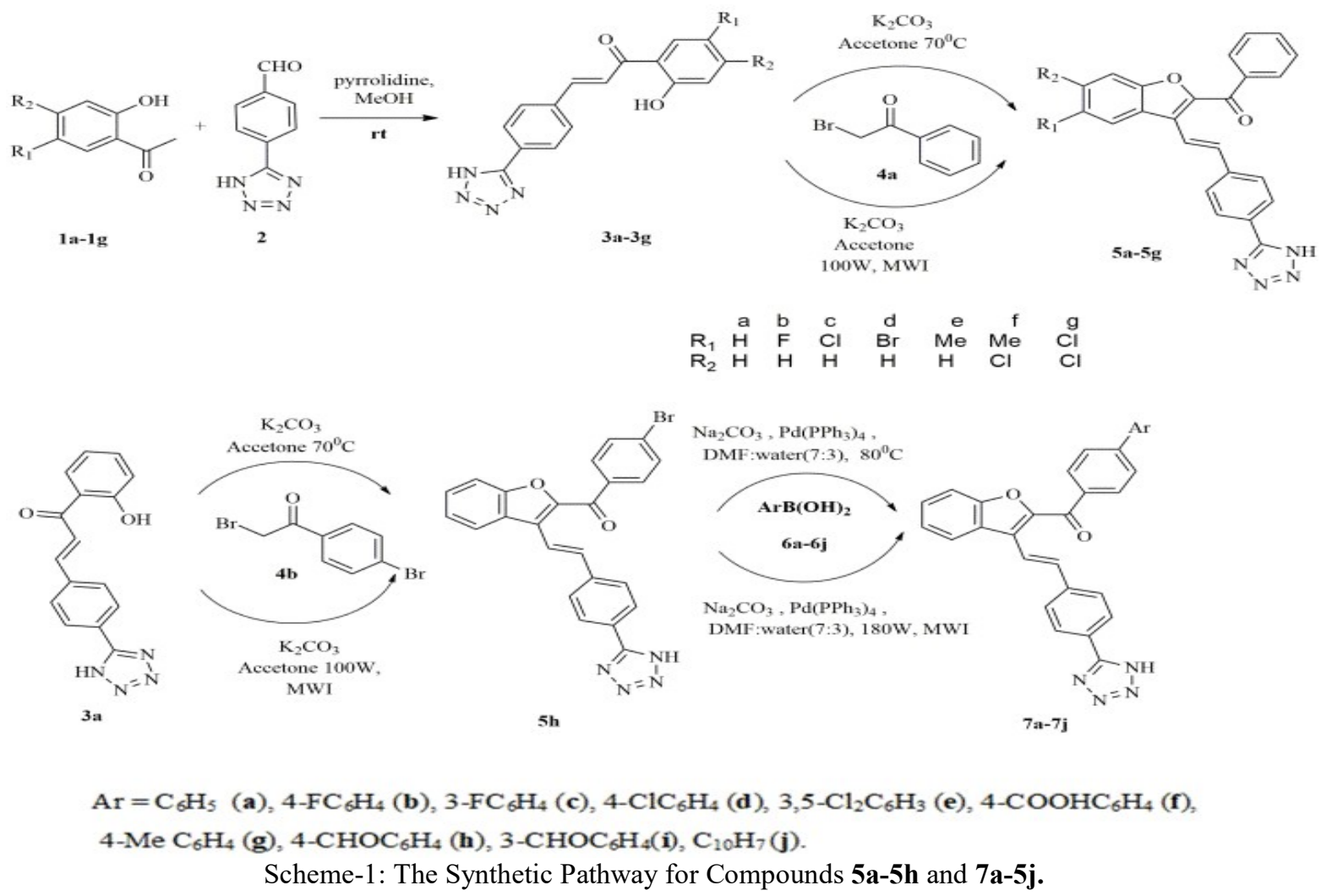

Scheme-1: The Synthetic Pathway for Compounds $\mathbf{5 a - 5 h}$ and $\mathbf{7 a - 5 j}$.

Table-1: The Comparison of Yields and Reaction Times of the Synthesized Compounds $\mathbf{5 a}-\mathbf{5 h}$ and $\mathbf{7 a}-\mathbf{7} \mathbf{j}$

\begin{tabular}{|c|c|c|c|c|c|c|c|c|c|}
\hline \multirow{2}{*}{ Compounds } & \multicolumn{2}{|c|}{$\begin{array}{l}\text { Conventional } \\
\text { Heating }\end{array}$} & \multicolumn{2}{|c|}{$\begin{array}{l}\text { Microwave } \\
\text { Irradiation }\end{array}$} & \multirow{2}{*}{ Compounds } & \multicolumn{2}{|c|}{$\begin{array}{c}\text { Conventional } \\
\text { Heating }\end{array}$} & \multicolumn{2}{|c|}{$\begin{array}{l}\text { Microwave } \\
\text { Irradiation }\end{array}$} \\
\hline & $\begin{array}{c}\text { Time } \\
(\mathrm{hr})\end{array}$ & $\begin{array}{l}\text { Yield } \\
(\%)\end{array}$ & $\begin{array}{l}\text { Time } \\
\text { (min) }\end{array}$ & $\begin{array}{l}\text { Yield } \\
(\%)\end{array}$ & & $\begin{array}{c}\text { Time } \\
(\mathrm{hr})\end{array}$ & $\begin{array}{c}\text { Yield } \\
(\%)\end{array}$ & $\begin{array}{l}\text { Time } \\
\text { (min) }\end{array}$ & $\begin{array}{l}\text { Yield } \\
(\%)\end{array}$ \\
\hline $5 a$ & 8 & 60 & 20 & 80 & $7 b$ & 7 & 62 & 21 & 76 \\
\hline $5 b$ & 6 & 65 & 22 & 72 & $7 c$ & 6 & 63 & 23 & 73 \\
\hline $5 c$ & 8 & 62 & 23 & 75 & $7 d$ & 8 & 50 & 20 & 80 \\
\hline 5d & 9 & 63 & 20 & 73 & $7 e$ & 9 & 62 & 23 & 79 \\
\hline $5 e$ & 9 & 50 & 20 & 76 & $7 f$ & 9 & 65 & 20 & 80 \\
\hline $5 f$ & 8 & 62 & 23 & 80 & $7 g$ & 8 & 60 & 23 & 74 \\
\hline $5 g$ & 7 & 65 & 20 & 72 & $7 \mathrm{~h}$ & 7 & 65 & 20 & 75 \\
\hline $5 \mathrm{~h}$ & 8 & 61 & 20 & 78 & $7 \mathbf{i}$ & 8 & 62 & 23 & 78 \\
\hline $7 a$ & 8 & 69 & 23 & 75 & $7 \mathbf{j}$ & 7 & 63 & 21 & 80 \\
\hline
\end{tabular}


RASĀYAN J. Chem.

Vol. 13 | No. 1 |601 - 609| January - March | 2020

We carefully studied for the Suzuki coupling reaction with $\mathrm{Pd}\left(\mathrm{PPh}_{3}\right)_{4}$ as catalyst and $\mathrm{Na}_{2} \mathrm{CO}_{3}$ as a base, from the screening studies Table-2. Finally, its confirmed the enhancement of the amount of catalyst $\left(\operatorname{Pd}\left(\mathrm{PPh}_{3}\right)_{4}\right)$ to obtain better yield. We successfully achieved, $15 \mathrm{~mol} \%$ of $\mathrm{Pd}\left(\mathrm{PPh}_{3}\right)_{4}$ as an optimal condition to get $80 \%$ of desired compounds (7a)

Table-2: Screening Studies for the Catalyst Loading.

\begin{tabular}{c|c|c|c|c}
\hline \multirow{2}{*}{$\begin{array}{c}\mathrm{Pd}\left(\mathrm{PPh}_{3}\right)_{4} \\
(\mathrm{~mol} \mathrm{\%})\end{array}$} & \multicolumn{2}{|c|}{ Conventional Heating } & \multicolumn{2}{c}{ Microwave Irradiation } \\
\cline { 2 - 5 } & Time (hr) & Yield (\%) & Time (min) & Yield (\%) \\
\hline 1 & 7 & 10 & 20 & 18 \\
\hline 4 & 7 & 12 & 20 & 16 \\
\hline 8 & 7 & 20 & 20 & 28 \\
\hline 12 & 7 & 25 & 20 & 40 \\
\hline $\mathbf{1 5}$ & $\mathbf{7}$ & $\mathbf{6 7}$ & $\mathbf{2 0}$ & $\mathbf{8 0}$ \\
\hline
\end{tabular}

The optimization of the reaction conditions was carried out by using different solvents and solvent mixtures (by using water as a co-solvent) and the results were depicted in Table-3. From these studies, it is clear that the tested solvents gave poor yields $(38-65 \%)$ when the reaction was carried out without water. But, when we added water as a co-solvent in different proportions the yield of the product was increased. Among them, DMF/Water (7/3) is found to be a better solvent mixture for further studies.

Table-3: The Effect of Different Solvents on the Suzuki Coupling Reaction 7a.

\begin{tabular}{c|c|c|c|c|c}
\hline \multirow{2}{*}{ Entry } & \multirow{2}{*}{ Solvent } & \multicolumn{2}{|c|}{ Conventional Heating } & \multicolumn{2}{|c}{ MWI } \\
\cline { 3 - 6 } & & Time (hr) & Yield (\%) & Time (min) & Yield (\%) \\
\hline 1 & DMF & 7 & 62 & 20 & 65 \\
\hline 2 & Dioxane & 7 & 52 & 20 & 56 \\
\hline 3 & NMP & 7 & 20 & 20 & 38 \\
\hline 4 & DMSO & 7 & 25 & 20 & 40 \\
\hline 5 & EtOH & 7 & 42 & 25 & 46 \\
\hline 6 & DMF / $\mathrm{H}_{2} \mathrm{O}(6: 4)$ & 7 & 65 & 25 & 69 \\
\hline 7 & DMF / $\mathrm{H}_{2} \mathrm{O}(7: 3)$ & 7 & 74 & 20 & 79 \\
\hline 8 & DMF / $\mathrm{H}_{2} \mathrm{O}(1: 1)$ & 7 & 64 & 22 & 58 \\
\hline
\end{tabular}

\section{Experimental Procedure for the Synthesis of Compounds 5a-5h}

\section{Conventional Heating Method}

To a solution of compounds 3a-3g ( $1 \mathrm{mmol})$, different substituted phenacyl bromides $4 \mathbf{a}, \mathbf{4 b}(1 \mathrm{mmol})$ and anhydrous $\mathrm{K}_{2} \mathrm{CO}_{3}(3 \mathrm{mmol})$ in dry acetone were stirred at $70^{\circ} \mathrm{C}$ for $6-8 \mathrm{hr}$. The completion of the reaction was checked by TLC. The resulting reaction mixture was poured in ice-cold water and the precipitate formed was filtered, washed with water, dried in air and purified by column chromatography (using 40 $60 \%$ petroleum ether/ethyl acetate) to afford desired compounds $\mathbf{5 a - 5 h}$.

\section{Microwave Irradiation Method:}

To a solution of compounds $\mathbf{3 a - 3} \mathbf{g}$ ( $1 \mathrm{mmol})$, different substituted phenacyl bromides $4 \mathbf{a}, \mathbf{4 b}(1 \mathrm{mmol})$ and anhydrous $\mathrm{K}_{2} \mathrm{CO}_{3}(3 \mathrm{mmol})$ in dry acetone were irradiated in the microwave at $100 \mathrm{~W}$ for $20-23 \mathrm{~min}$. The completion of the reaction was checked by TLC. The resulting reaction mixture was poured in ice-cold water and the precipitate formed was filtered, washed with water, dried in air and purified by column chromatography (using 40-60\% petroleum ether/ethyl acetate) to afford desired compounds $\mathbf{5 a - 5 h}$.

\section{Characterization of Compounds 5a-5h}

(E)-(3-(4-(1H-tetrazol-5-yl)styryl)benzofuran-2-yl)(phenyl)methanone (5a): $\mathrm{mp} \quad 250-252^{\circ} \mathrm{C} . \quad \mathrm{IR}$ spectrum, $v, \mathrm{~cm}^{-1}: 3143,2922,1627$ (CO), $1265 ;{ }^{1} \mathrm{H}$ NMR spectrum, $\delta$, ppm: 7.36-7.52 m (10H, Ar-H), $7.74 \mathrm{~d}(2 \mathrm{H}, \mathrm{Ar}-\mathrm{H}, J=8.5 \mathrm{~Hz}), 7.84 \mathrm{~d}(1 \mathrm{H}, \mathrm{H \alpha}, J=16.8 \mathrm{~Hz}), 8.01 \mathrm{~d}(2 \mathrm{H}, \mathrm{Ar}-\mathrm{H}, J=8.5 \mathrm{~Hz},) .{ }^{13} \mathrm{C}$ NMR spectrum, $\delta_{\mathrm{C}}$, ppm: 119.2, 119.6, 119.9, 122.7, 124.9, 125.3, 126.1, 127.0, 127.7, 128.4, 128.7, 129.5, 
129.8, 132.9, 134.7, 137.6, 139.5, 146.4, 153.0, 185.7. Mass spectrum, $\mathrm{m} / z$ (Irel, \%): 392 [M+H]; Found, \%: C 73.46; H 4.11; N 14.28. $\mathrm{C}_{24} \mathrm{H}_{16} \mathrm{~N}_{4} \mathrm{O}_{2}$. Calculated, \%: C 73.49; H 4.14; N 14.35.

(E)-(3-(4-(1H-tetrazol-5-yl)styryl)-5-fluoro benzofuran-2-yl)(phenyl)methanone (5b):mp 248-112 ${ }^{\circ} \mathrm{C}$. IR spectrum, $v, \mathrm{~cm}^{-1}: 3157,2941,1622$ (CO), $1267 ;{ }^{1} \mathrm{H}$ NMR spectrum, $\delta$, ppm: 7.50-7.66 m (9H, Ar-H), $7.79 \mathrm{~d}(2 \mathrm{H}, \mathrm{Ar}-\mathrm{H}, J=8.5 \mathrm{~Hz}), 7.86 \mathrm{~d}(1 \mathrm{H}, \mathrm{H \alpha}, J=16.8 \mathrm{~Hz}), 8.08 \mathrm{~d}(2 \mathrm{H}, \mathrm{Ar}-\mathrm{H}, J=8.5 \mathrm{~Hz}) .{ }^{13} \mathrm{C}$ NMR spectrum, $\delta_{\mathrm{C}}$, ppm: $113.7,114.1,119.2,119.6,122.7,124.9,125.3,126.1,126.9,127.8,128.4,129.5$, $129.8,131.3,132.9,134.7,137.6,139.5,146.4,148.6,153.0,185.7$. Mass spectrum, $\mathrm{m} / \mathrm{z}$ (Irel, \%): 411 $[\mathrm{M}+\mathrm{H}]$; Found, \%: C 70.24; H 3.68; F 4.63; N 13.65. $\mathrm{C}_{24} \mathrm{H}_{15} \mathrm{FN}_{4} \mathrm{O}_{2}$. Calculated, \%: C 70.29; H 3.77; F 4.68; N 13.69 .

(E)-(3-(4-(1H-tetrazol-5-yl)styryl)-5-chloro benzofuran-2-yl)(phenyl)methanone (5c): mp 266-268 ${ }^{\circ} \mathrm{C}$. IR spectrum, $v, \mathrm{~cm}^{-1}: 3076,2941,1622(\mathrm{CO}), 1267 ;{ }^{1} \mathrm{H}$ NMR spectrum, $\delta$, ppm: 7.45-7.57 m (9H, Ar-H), $7.78 \mathrm{~d}(2 \mathrm{H}, \mathrm{Ar}-\mathrm{H}, J=7.8 \mathrm{~Hz}), 7.88 \mathrm{~d}(1 \mathrm{H}, \mathrm{H \alpha}, J=16.8 \mathrm{~Hz}), 8.10 \mathrm{~d}(2 \mathrm{H}, \mathrm{Ar}-\mathrm{H}, J=7.8 \mathrm{~Hz}) .{ }^{13} \mathrm{C}$ NMR spectrum, $\delta_{\mathrm{C}}$, ppm: 113.7, 119.2, 119.6, 122.7, 124.9, 126.1, 126.9, 127.7, 128.4, 129.5, 129.8, 131.3, 132.9, 134.7, 137.6, 139.5, 146.4, 148.6, 153.0, 185.7. Mass spectrum, $\mathrm{m} / z$ (Irel, \%): 427 [M+H]; Found, \%: C 67.53; H 3.54; $\mathrm{Cl} 8.31 ; \mathrm{N}$ 13.13. $\mathrm{C}_{24} \mathrm{H}_{15} \mathrm{ClN}_{4} \mathrm{O}_{2}$. Calculated, \%: C 67.58; H 3.57; $\mathrm{Cl} 8.34 ; \mathrm{N} 13.19$.

(E)-(3-(4-(1H-tetrazol-5-yl)styryl)-5-bromo benzofuran-2-yl)(phenyl)methanone (5d): mp 248-250 ${ }^{\circ} \mathrm{C}$. IR spectrum, $v, \mathrm{~cm}^{-1}: 3178,2927,1597(\mathrm{CO}), 1261 ;{ }^{1} \mathrm{H}$ NMR spectrum, $\delta$, ppm: 7.47-7.65 m (9H, Ar-H), $7.81 \mathrm{~d}(2 \mathrm{H}, \mathrm{Ar}-\mathrm{H}, J=7.2 \mathrm{H}), 7.87 \mathrm{~d}(1 \mathrm{H}, \mathrm{H \alpha}, J=16.8 \mathrm{~Hz}), 8.08 \mathrm{~d}(2 \mathrm{H}, \mathrm{Ar}-\mathrm{H}, J=7.2 \mathrm{~Hz}) .{ }^{13} \mathrm{C}$ NMR spectrum, $\delta_{\mathrm{C}}$, ppm: 113.7, 119.2, 119.6, 122.7, 125.0, 125.3, 126.1, 126.3, 127.0, 127.7, 128.4, 129.5, $129.8,132.9,134.7,137.6,139.5,146.4,153.0,185.7$. Mass spectrum, $m / z$ (Irel, \%): 471 [M+H]; Found, \%: C 61.16; H 3.21; $\mathrm{Br} 16.95 ; \mathrm{N}$ 11.89. $\mathrm{C}_{24} \mathrm{H}_{15} \mathrm{BrN}_{4} \mathrm{O}_{2}$. Calculated, \%: C 61.19; H 3.26; $\mathrm{Br} 16.90 ; \mathrm{N}$ 11.87.

(E)-(3-(4-(1H-tetrazol-5-yl)styryl)-5-methyl benzofuran-2-yl)(phenyl)methanone (5e): mp 234$236^{\circ} \mathrm{C}$. IR spectrum, $v, \mathrm{~cm}^{-1}: 3143,2941,1621(\mathrm{CO}), 1267 ;{ }^{1} \mathrm{H}$ NMR spectrum, $\delta$, ppm: $3.22 \mathrm{~s}(3 \mathrm{H}$, $\left.\mathrm{CH}_{3}\right), 7.45-7.66 \mathrm{~m}(9 \mathrm{H}, \mathrm{Ar}-\mathrm{H}), 7.92 \mathrm{~d}(2 \mathrm{H}, \mathrm{Ar}-\mathrm{H}, J=8.53 \mathrm{~Hz}), 7.99 \mathrm{~d}(1 \mathrm{H}, \mathrm{H \alpha}, J=16.8 \mathrm{~Hz}), 8.21 \mathrm{~d}(2 \mathrm{H}$, $\mathrm{Ar}-\mathrm{H}, J=8.5 \mathrm{~Hz}) .{ }^{13} \mathrm{C}$ NMR spectrum, $\delta_{\mathrm{C}}$, ppm: 22.4, 114.0, 119.5, 119.9, 123.0, 125.2, 125.6, 126.4, $127.2,128.0,128.7,129.8,130.0,133.2,134.9,137.9,139.8,146.7,148.9,153.3,185.9$. Mass spectrum, $m / z$ (Irel, \%): 407 [M+H]; Found, \%: C 73.88; H 4.46; N 13.78. $\mathrm{C}_{25} \mathrm{H}_{18} \mathrm{~N}_{4} \mathrm{O}_{2}$. Calculated, \%: C 73.92; $\mathrm{H}$ 4.42; N 13.74 .

(E)-(3-(4-(1H-tetrazol-5-yl)styryl)-5-chloro-6-methyl benzofuran-2-yl)(phenyl)methanone (5f): $\mathrm{mp}$ 220-222 ${ }^{\circ} \mathrm{C}$. IR spectrum, $v, \mathrm{~cm}^{-1}: 3076,2941,1622$ (CO), 1267; ${ }^{1} \mathrm{H}$ NMR spectrum, $\delta$, ppm: $3.62 \mathrm{~s}(3 \mathrm{H}$, $\left.\mathrm{CH}_{3}\right), 7.45-7.64 \mathrm{~m}(8 \mathrm{H}, \mathrm{Ar}-\mathrm{H}), 7.84 \mathrm{~d}(2 \mathrm{H}, \mathrm{Ar}-\mathrm{H}, J=7.28 \mathrm{~Hz}), 7.95 \mathrm{~d}(1 \mathrm{H}, \mathrm{H \alpha}, J=16.5 \mathrm{~Hz}), 8.23 \mathrm{~d}(2 \mathrm{H}$, $\mathrm{Ar}-\mathrm{H}, J=7.2 \mathrm{~Hz}) .{ }^{13} \mathrm{C}$ NMR spectrum, $\delta_{\mathrm{C}}$, ppm: 25.0, 109.0, 114.5, 114.9, 118.0, 120.3, 120.6, 121.4, 122.2, 123.0, 123.7, 124.8, 125.1, 128.2, 130.0, 132.9, 134.8, 141.7, 143.9, 148.3, 181.0. Mass spectrum, $m / z$ (Irel, \%): $441[\mathrm{M}+\mathrm{H}]$; Found, \%: C 68.11; H 3.89; $\mathrm{Cl} 8.04 ; \mathrm{N} 12.71 . \mathrm{C}_{25} \mathrm{H}_{17} \mathrm{ClN}_{4} \mathrm{O}_{2}$. Calculated, \%: C 68.17 ; $3.97 ; \mathrm{Cl} 8.12 ; \mathrm{N} 12.77$.

(E)-(3-(4-(1H-tetrazol-5-yl)styryl)-5,6-dichlorobenzofuran-2-yl)(phenyl)methanone $\quad(5 \mathrm{~g}): \quad \mathrm{mp} \quad 210$ $212^{\circ} \mathrm{C}$. IR spectrum, $v, \mathrm{~cm}^{-1}: 3143,2941,1627$ (CO), $1265 ;{ }^{1} \mathrm{H}$ NMR spectrum, $\delta$, ppm: 7.50-7.58 m $(8 \mathrm{H}, \mathrm{Ar}-\mathrm{H}), 7.81 \mathrm{~d}(2 \mathrm{H}, \mathrm{Ar}-\mathrm{H}, J=7.2 \mathrm{~Hz}), 7.89 \mathrm{~d}(1 \mathrm{H}, \mathrm{H \alpha}, J=16.5 \mathrm{~Hz}), 8.17 \mathrm{~d}(2 \mathrm{H}, \mathrm{Ar}-\mathrm{H}, J=7.2 \mathrm{~Hz}) .{ }^{13} \mathrm{C}$ NMR spectrum, $\delta_{\mathrm{C}}$, ppm: 119.1, 119.7, 121.3, 125.0, 125.8, 126.3, 127.0, 127.7, 128.1, 128.4, 129.5, 129.9, 133.1, 134.6, 137.2, 139.4, 146.4, 148.7, 149.1, 184.9. Mass spectrum, $m / z$ (Irel, \%): $461[\mathrm{M}+\mathrm{H}]$; Found, \%: C 62.49; H 3.06; Cl 15.37; N 12.15. $\mathrm{C}_{24} \mathrm{H}_{14} \mathrm{Cl}_{2} \mathrm{~N}_{4} \mathrm{O}_{2}$. Calculated, \%: C 62.44; H 3.16; $\mathrm{Cl} 15.39$; N 12.18 .

(E)-(3-(4-(1H-tetrazol-5-yl)styryl)benzofuran-2-yl)(4-bromophenyl)methanone (5h): mp 260-262 ${ }^{\circ} \mathrm{C}$. IR spectrum, $v, \mathrm{~cm}^{-1}: 3167,2924,1662(\mathrm{CO}), 1282 ;{ }^{1} \mathrm{H}$ NMR spectrum, $\delta$, ppm: 7.45-7.57 m (7H, Ar-H), 7.62-7.66 m (2H, Ar-H), $7.81 \mathrm{~d}(2 \mathrm{H}, \mathrm{Ar}-\mathrm{H}, J=7.8 \mathrm{~Hz}), 7.88 \mathrm{~d}(1 \mathrm{H}, \mathrm{H \alpha}, J=16.8 \mathrm{~Hz}), 8.10 \mathrm{~d}(2 \mathrm{H}, \mathrm{Ar}-\mathrm{H}, J$ $=7.8 \mathrm{~Hz}) .{ }^{13} \mathrm{C}$ NMR spectrum, $\delta_{\mathrm{C}}$, ppm: $113.7,117.4,119.2,125.0,125.3,126.2,127.0,127.8,128.4$, 
128.4, 129.5, 129.8, 131.3, 132.9, 134.7, 137.6, 139.5, 146.5, 148.6, 153.0, 185.7. Mass spectrum, $\mathrm{m} / \mathrm{z}$ (Irel, \%): $471[\mathrm{M}+\mathrm{H}]$; Found, \%: C 61.16; H 3.21; $\mathrm{Br} 16.95 ; \mathrm{N}$ 11.89. $\mathrm{C}_{24} \mathrm{H}_{15} \mathrm{Br}_{2} \mathrm{~N}_{4} \mathrm{O}_{2}$. Calculated, \%: C 61.19; H 3.24; Br 16.99; N 11.85.

\section{General Experimental Procedure 7a-7j \\ Conventional Heating Method}

A degassed solution of $\mathbf{5 h}(1.0 \mathrm{mmol})$, arylboronic acid $\mathbf{6 a}-\mathbf{6 j}(1.3 \mathrm{mmol}), \mathrm{Na}_{2} \mathrm{CO}_{3}(3.00 \mathrm{mmol})$, $\mathrm{Pd}\left(\mathrm{PPh}_{3}\right)_{4}(0.10 \mathrm{mmol})$ and solution of DMF/water (ratio 6:4) was introduced into a round-bottomed flask and heated it at $80^{\circ} \mathrm{C}$ for $6-9 \mathrm{hr}$ with constant stirring under nitrogen atmosphere. The resulting solution was diluted with ice-cold water and was extracted with ethyl acetate; the combined organic layers were washed with brine, dried over $\mathrm{Na}_{2} \mathrm{SO}_{4}$. The solvent was removed under reduced pressure and the crude thus obtained was purified using column chromatography to afford titled compounds.

\section{Microwave Irradiation Method}

A degassed mixture of $\mathbf{5 h}(1.0 \mathrm{mmol})$, aryl boronic acid $\mathbf{6 a - 6 j}(1.3 \mathrm{mmol}), \mathrm{Na}_{2} \mathrm{CO}_{3}(3.00 \mathrm{mmol})$, $\mathrm{Pd}\left(\mathrm{PPh}_{3}\right)_{4}(0.10 \mathrm{mmol})$ and a mixture of DMF/water (ratio 6:4) in a sealed pressure tube. This was introduced into CEM discover microwave reaction vessel equipped with a magnetic stirrer. The vessel was sealed and then placed into the microwave cavity. Initial microwave irradiation of $180 \mathrm{~W}$ was used, the temperature is ramped from room temperature to the desired $80^{\circ} \mathrm{C}$ temperature. Once this was reached the reaction mixture was heated at this temperature for the appropriate time. The resulting solution was diluted with ice water and was extracted with ethyl acetate; the combined organic layers were washed with brine, dried over $\mathrm{Na}_{2} \mathrm{SO}_{4}$. The solvent was removed under reduced pressure and the crude thus obtained was purified using column chromatography to afford titled compounds.

\section{Characterization of Compounds $7 \mathbf{a}-7 \mathbf{j}$}

(E)-(3-(4-(1H-tetrazol-5-yl) styryl)benzofuran-2-yl)([1,1'-biphenyl]-4-yl)methanone (7a): $\mathrm{mp} \quad 284-$ $286^{\circ} \mathrm{C}$. IR spectrum, $v, \mathrm{~cm}^{-1}: 3072,2922,1740(\mathrm{CO}), 1630,1232 ;{ }^{1} \mathrm{H}$ NMR spectrum, $\delta$, ppm: 7.30-7.34 $\mathrm{m}(1 \mathrm{H}, \mathrm{Ar}-\mathrm{H}), 7.42-7.58 \mathrm{~m}(9 \mathrm{H}, \mathrm{Ar}-\mathrm{H}), 7.68-7.72 \mathrm{~m}(4 \mathrm{H}, \mathrm{Ar}-\mathrm{H}), 7.76-7.81 \mathrm{~m}(2 \mathrm{H}, \mathrm{Ar}-\mathrm{H}), 7.98 \mathrm{~d}(1 \mathrm{H}$, $\mathrm{H \alpha}, J=16.6 \mathrm{~Hz}), 8.21 \mathrm{~d}(2 \mathrm{H}, \mathrm{Ar}-\mathrm{H}, J=8.5 \mathrm{~Hz}) .{ }^{13} \mathrm{C}$ NMR spectrum, $\delta_{\mathrm{C}}, \mathrm{ppm}: 112.7,119.2,120.4,123.1$, $124.3,124.8,125.0,126.0,126.1,127.0,127.3,127.7,129.0,129.5,130.5,135.0,136.7,139.5,145.5$, 146.3, 147.0, 154.8, 185.4. Mass spectrum, $m / z$ (Irel, \%): 369 [M+H]; Found, \%: C 76.91; H 4.30; N 11.96. $\mathrm{C}_{30} \mathrm{H}_{20} \mathrm{~N}_{4} \mathrm{O}_{2}$. Calculated, \%: C 76.91; H 4.30; N 11.96 .

(E)-(3-(4-(1H-tetrazol-5-yl) styryl)benzofuran-2-yl)(4'-fluoro-[1,1'-biphenyl]-4-yl)methanone (7b): $\mathrm{mp}$ 240-242 ${ }^{\circ} \mathrm{C}$. IR spectrum, $v, \mathrm{~cm}^{-1}: 3071,2922,1741$ (CO), 1631, 1235; ${ }^{1} \mathrm{H}$ NMR spectrum, $\delta$, ppm: 7.16$7.21 \mathrm{~m}(2 \mathrm{H}, \mathrm{Ar}-\mathrm{H}), 7.43-7.66 \mathrm{~m}(9 \mathrm{H}, \mathrm{Ar}-\mathrm{H}), 7.73 \mathrm{~d}(2 \mathrm{H}, \mathrm{Ar}-\mathrm{H}, J=8.2 \mathrm{~Hz}), 7.79 \mathrm{~d}(2 \mathrm{H}, \mathrm{Ar}-\mathrm{H}, J=7.5 \mathrm{~Hz})$, $7.98 \mathrm{~d}(1 \mathrm{H}, \mathrm{H \alpha}, J=16.6 \mathrm{~Hz}), 8.20 \mathrm{~d}(2 \mathrm{H}, \mathrm{Ar}-\mathrm{H}, J=8.5 \mathrm{~Hz}) .{ }^{13} \mathrm{C}$ NMR spectrum, $\delta_{\mathrm{C}}, \mathrm{ppm}: 112.7,115.8$, $116.1,119.2,120.4,123.1,124.3,124.9,126.0,126.1,126.9,127.7,128.9,129.1,129.5,130.5,135.0$, 136.1, 136.1, 136.7, 139.5, 144.4, 146.3, 147.9, 154.8, 185.3. Mass spectrum, $m / z$ (Irel, \%): 487 [M+H]; Found, \%: C 74.06; H 3.94; F 3.91; N 11.52. $\mathrm{C}_{30} \mathrm{H}_{19} \mathrm{FN}_{4} \mathrm{O}_{2}$. Calculated, \%: C 74.16; H 3.90; F 3.95; N 11.58 .

(E)-(3-(4-(1 H-tetrazol-5-yl)styryl)benzofuran-2-yl)(3'-fluoro-[1,1'-biphenyl]-4-yl)methanone (7c): mp 210-212 ${ }^{\circ} \mathrm{C}$. IR spectrum, $v, \mathrm{~cm}^{-1}: 3135,2924,1742$ (CO), 1598, 1226; ${ }^{1} \mathrm{H}$ NMR spectrum, $\delta$, ppm: 7.18-7.20 m (1H, Ar-H), 7.33-7.58 m (12H, Ar-H), $7.69 \mathrm{~d}(2 \mathrm{H}, \mathrm{Ar}-\mathrm{H}, J=8.2 \mathrm{~Hz}), 7.74 \mathrm{~d}(1 \mathrm{H}, \mathrm{Ar}-\mathrm{H}, J$ $=16.5 \mathrm{~Hz}), 7.99 \mathrm{~d}(2 \mathrm{H}, \mathrm{Ar}-\mathrm{H}, J=8.2 \mathrm{~Hz}) .{ }^{13} \mathrm{C}$ NMR spectrum, $\delta_{\mathrm{C}}, \mathrm{ppm}: 112.6,119.2,120.1,123.2,124.3$, $125.0,125.3,125.8,126.0,126.0,126.9,127.7,128.6,129.5,130.0,130.7,131.7,131.9,134.9,136.6$, 139.5, 146.3, 147.4, 152.8, 154.8, 184.6. Mass spectrum, $m / z$ (Irel, \%): 487 [M+H]; Found, \%: C 74.06; H 3.94; F 3.91; $\mathrm{N}$ 11.52. $\mathrm{C}_{30} \mathrm{H}_{19} \mathrm{FN}_{4} \mathrm{O}_{2}$. Calculated, \%: C 74.10; H 3.99; F 3.95; N 11.57.

(E)-(3-(4-(1H-tetrazol-5-yl) styryl)benzofuran-2-yl)(4'-chloro-[1,1'-biphenyl]-4-yl)methanone (7d): mp 246-246 ${ }^{\circ} \mathrm{C}$. IR spectrum, $v, \mathrm{~cm}^{-1}$ : 2923, 1738(CO), 1630, 1230; ${ }^{1} \mathrm{H}$ NMR spectrum, $\delta$, ppm: 7.18-7.20 m 
$(1 \mathrm{H}, \mathrm{Ar}-\mathrm{H}), 7.31-7.35 \mathrm{~m}(1 \mathrm{H}, \mathrm{Ar}-\mathrm{H}), 7.43-7.60 \mathrm{~m}(5 \mathrm{H}, \mathrm{Ar}-\mathrm{H}), 7.50-7.72 \mathrm{~m}(4 \mathrm{H}, \mathrm{Ar}-\mathrm{H}), 7.81 \mathrm{~d}(2 \mathrm{H}, \mathrm{Ar}-\mathrm{H}$, $J=8.0 \mathrm{~Hz}), 7.96 \mathrm{~d}(1 \mathrm{H}, \mathrm{H \alpha}, J=16.3 \mathrm{~Hz}), 7.95 \mathrm{~d}(2 \mathrm{H}, \mathrm{Ar}-\mathrm{H}, J=8.2 \mathrm{~Hz}), 8.13 \mathrm{~d}(2 \mathrm{H}, \mathrm{Ar}-\mathrm{H}, J=8.0 \mathrm{~Hz}) .{ }^{13} \mathrm{C}$ NMR spectrum, $\delta_{\mathrm{C}}$, ppm: $105.9,109.1,112.4,113.4,116.4,117.5,118.4,119.1,119.2,119.3,120.1$, $120.5,120.9,121.1,121.7,122.7,123.5,123.8,129.0,130.9,132.7,136.9,139.5,148.0,185.0$ Mass spectrum, $m / z$ (Irel, \%): $503[\mathrm{M}+\mathrm{H}]$; Found, \%: C 71.64; $\mathrm{H} 3.81 ; \mathrm{Cl} 7.05 ; \mathrm{N} 11.14 . \mathrm{C}_{30} \mathrm{H}_{19} \mathrm{ClN}_{4} \mathrm{O}_{2}$. Calculated, \%: C 71.69; H 3.88; Cl 7.04; N 11.17.

(E)-(3-(4-(1H-tetrazol-5-yl)styryl)benzofuran-2-yl)(2',4'-dichloro-[1,1'-biphenyl]-4-yl)methanone (7e): mp 248-250 ${ }^{\circ} \mathrm{C}$. IR spectrum, $v, \mathrm{~cm}^{-1}: 3161,2970,1740(\mathrm{CO}), 1598,1233 ;{ }^{1} \mathrm{H}$ NMR spectrum, $\delta$, ppm: 7.31-7.35 t (1H, Ar-H), 7.40-7.62 m (9H, Ar-H), 7.71 d (2H, Ar-H, $J=8.5 \mathrm{~Hz}), 7.80 \mathrm{~d}(2 \mathrm{H}, \mathrm{Ar}-\mathrm{H}, J=8.5$ $\mathrm{Hz}), 8.00 \mathrm{~d}(1 \mathrm{H}, \mathrm{Ar}-\mathrm{H}, J=16.8 \mathrm{~Hz}), 8.22 \mathrm{~d}(2 \mathrm{H}, \mathrm{Ar}-\mathrm{H}, J=8.5 \mathrm{~Hz}) .{ }^{13} \mathrm{C}$ NMR spectrum, $\delta_{\mathrm{C}}, \mathrm{ppm}: 112.7$, $119.2,120.3,123.2,124.4,124.8,125.0,125.2,126.0,126.2,126.9,127.0,127.6,127.7,128.1,128.6$, 129.5, 130.6, 135.6, 137.7, 139.5, 142.5, 143.0, 147.7, 154.8, 179.7. Mass spectrum, $\mathrm{m} / \mathrm{z}$ (Irel, \%): 537 $[\mathrm{M}+\mathrm{H}]$; Found, \%: C 67.05; H 3.38; Cl 13.19; N 10.43. $\mathrm{C}_{30} \mathrm{H}_{18} \mathrm{Cl}_{2} \mathrm{~N}_{4} \mathrm{O}_{2}$. Calculated, \%: C 67.10; H 3.34; Cl 14.11; N 11.03.

(E)-4'-(3-(4-(1H-tetrazol-5-yl)styryl)benzofuran-2-carbonyl)-[1,1'-biphenyl]-4-carboxylic acid (7f): $\mathrm{mp}$ 240-242 ${ }^{\circ} \mathrm{C}$. IR spectrum, $v, \mathrm{~cm}^{-1}: 3159,2956,1720$ (CO), 1548, 1231; ${ }^{1} \mathrm{H}$ NMR spectrum, $\delta$, ppm: 7.69$7.44 \mathrm{~m}(9 \mathrm{H}, \mathrm{Ar}-\mathrm{H}), 7.83-7.73 \mathrm{~m}(4 \mathrm{H}, \mathrm{Ar}-\mathrm{H}), 7.82 \mathrm{~d}(2 \mathrm{H}, \mathrm{Ar}-\mathrm{H}, J=7.8 \mathrm{~Hz}),, 7.97 \mathrm{~d}(1 \mathrm{H}, \mathrm{Ar}-\mathrm{H}, J=16.56$ $\mathrm{Hz}), 8.00 \mathrm{~d}(2 \mathrm{H}, \mathrm{Ar}-\mathrm{H}, J=8.3 \mathrm{~Hz}), 8.13 \mathrm{~d}(1 \mathrm{H}, \mathrm{Ar}-\mathrm{H}, J=8.0 \mathrm{~Hz}) .{ }^{13} \mathrm{C}$ NMR spectrum, $\delta \mathrm{C}$, ppm: 112.7 , $119.2,120.2,123.2,124.4,125.0,125.3,125.8,126.0,126.2,126.7,126.9,127.7,127.8,128.6,129.5$, 131.4, 131.7, 134.9, 136.7, 139.5, 146.4, 147.4, 147.8, 154.8, 180.5. Mass spectrum, $\mathrm{m} / \mathrm{z}$ (Irel, \%): 513[M+H]; Found, \%: C 72.65; H 3.93; N 10.93. $\mathrm{C}_{31} \mathrm{H}_{20} \mathrm{~N}_{4} \mathrm{O}_{3}$. Calculated, \%: C 72.64; H 3.90; N 10.99.

(E)-(3-(4-(1H-tetrazol-5-yl) styryl)benzofuran-2-yl)(4'-methyl-[1,1'-biphenyl]-4-yl)methanone (7g): $\mathrm{mp}$ 230-232 ${ }^{\circ} \mathrm{C}$. IR spectrum, $v, \mathrm{~cm}^{-1}: 3072,2923,1740$ (CO), 1627, 1231; ${ }^{1} \mathrm{H}$ NMR spectrum, $\delta$, ppm: $2.42 \mathrm{~s}$ $\left(3 \mathrm{H}, \mathrm{CH}_{3}\right), 7.18-7.20 \mathrm{~m}(1 \mathrm{H}, \mathrm{Ar}-\mathrm{H}), 7.28-7.33 \mathrm{~m}(2 \mathrm{H}, \mathrm{Ar}-\mathrm{H}), 7.42-7.63 \mathrm{~m}(8 \mathrm{H}, \mathrm{Ar}-\mathrm{H}), 7.75-7.79 \mathrm{~m}(4 \mathrm{H}$, $\mathrm{Ar}-\mathrm{H}), 7.96 \mathrm{~d}(1 \mathrm{H}, \mathrm{H \alpha}, J=16.6 \mathrm{~Hz}), 8.18 \mathrm{~d}(2 \mathrm{H}, \mathrm{Ar}-\mathrm{H}, J=8.3 \mathrm{~Hz}) .{ }^{13} \mathrm{C}$ NMR spectrum, $\delta_{\mathrm{C}}$, ppm: 22.0 , $112.7,119.1,120.4,123.1,124.2,124.7,124.9,126.1,126.8,127.2,127.7,128.3,129.5,130.5,136.4$, 137.0, 137.9, 138.2, 139.5, 145.4, 146.3, 148.0,154.8, 185.4. Mass spectrum, $m / z$ (Irel, \%): 483 [M+H]; Found, \%: C 77.16; H 4.60; N 11.61. $\mathrm{C}_{31} \mathrm{H}_{22} \mathrm{~N}_{4} \mathrm{O}_{2}$. Calculated, \%: C 77.06; H 4.66; N 11.64.

(E)-4'-(3-(4-(1H-tetrazol-5-yl)styryl)benzofuran-2-carbonyl)-[1,1'-biphenyl]-4-carbaldehyde (7h): $\mathrm{mp}$ 265-267 ${ }^{\circ} \mathrm{C}$. IR spectrum, $v, \mathrm{~cm}^{-1}: 3069,2926,1740$ (CO), 1602, 1216; ${ }^{1} \mathrm{H}$ NMR spectrum, $\delta$, ppm: $6.96 \mathrm{~d}$ $(3 \mathrm{H}, J=8.5, \mathrm{Ar}-\mathrm{H}), 7.20 \mathrm{t}(1 \mathrm{H}, \mathrm{Ar}-\mathrm{H}), 7.33 \mathrm{t}(1 \mathrm{H}, \mathrm{Ar}-\mathrm{H}), 7.73-7.86 \mathrm{~m}(10 \mathrm{H}, \mathrm{Ar}-\mathrm{H}), 8.00(\mathrm{~d}, 1 \mathrm{H}, \mathrm{Ar}-\mathrm{H}, J$ $=16.0 \mathrm{~Hz}), 8.24 \mathrm{~d}(2 \mathrm{H}, \mathrm{Ar}-\mathrm{H}, J=8.2 \mathrm{~Hz}),, 9.84 \mathrm{~s}(1 \mathrm{H}, \mathrm{CHO}) .{ }^{13} \mathrm{C}$ NMR spectrum, $\delta_{\mathrm{C}}$, ppm: 112.7, 115.9, $119.3,120.3,123.2,124.4,126.1,126.2,126.2,127.0,127.3,127.7,128.0,128.6,129.5,130.6,134.1$, 134.9, 135.8, 137.7, 139.5, 143.7, 146.4, 154.8, 191.8, 199.1, Mass spectrum, $m / z$ (Irel, \%): 497 [M+H]; Found, \%: C 74.99; $\mathrm{H}$ 4.06; $\mathrm{N}$ 11.28. $\mathrm{C}_{31} \mathrm{H}_{20} \mathrm{~N}_{4} \mathrm{O}_{3}$. Calculated, \%: C 74.94; H 4.11; N 11.29.

(E)-4'-(3-(4-(1H-tetrazol-5-yl)styryl)benzofuran-2-carbonyl)-[1,1'-biphenyl]-3-carbaldehyde (7i): $\mathrm{mp}$ 256-258 ${ }^{\circ} \mathrm{C}$. IR spectrum, $v, \mathrm{~cm}^{-1}: 3089,2925,1740$ (CO), 1602, 1231; ${ }^{1} \mathrm{H}$ NMR spectrum, $\delta$, ppm: 7.44$7.69 \mathrm{~m}(8 \mathrm{H}, \mathrm{Ar}-\mathrm{H}), 7.73-7.83 \mathrm{~m}(4 \mathrm{H}, \mathrm{Ar}-\mathrm{H}), 7.92-7.96 \mathrm{~m}(3 \mathrm{H}, \mathrm{Ar}-\mathrm{H}), 8.02 \mathrm{~d}(1 \mathrm{H}, \mathrm{Ar}-\mathrm{H}, J=16.5 \mathrm{~Hz})$, $8.24 \mathrm{~d}(2 \mathrm{H}, \mathrm{Ar}-\mathrm{H}, J=8.2 \mathrm{~Hz}), 10.12 \mathrm{~s}(1 \mathrm{H}, \mathrm{CHO}) .{ }^{13} \mathrm{C}$ NMR spectrum, $\delta_{\mathrm{C}}$, ppm: $110.6,117.1,118.2$, $121.1,122.3,123.0,124.1,125.0,125.5,125.7,126.2$, 126.4, 127.5, 127.7, 128.6, 131.0, 135.0, 137.4, 138.9, 141.7, 144.3,145.7, 152.8, 183.2, 190.0. Mass spectrum, $m / z$ (Irel, \%): 497 [M+H]; Found, \%: C 74.99; $\mathrm{H} 4.06 ; \mathrm{N} 11.28 . \mathrm{C}_{31} \mathrm{H}_{20} \mathrm{~N}_{4} \mathrm{O}_{3}$. Calculated, \%: C 74.94; $\mathrm{H} 4.11 ; \mathrm{N} 11.29$.

(E)-(3-(4-(1H-tetrazol-5-yl)styryl)benzofuran-2-yl)(4-(naphthalen-1-yl)phenyl)methanone (7j): $\mathrm{mp}$ 242-246 ${ }^{\circ} \mathrm{C}$. IR spectrum, $v, \mathrm{~cm}^{-1}: 3366,2924,1739$ (CO), 1598, 1229; ${ }^{1} \mathrm{H}$ NMR spectrum, $\delta$, ppm: 7.43$7.59 \mathrm{~m}(11 \mathrm{H}, \mathrm{Ar}-\mathrm{H}), 7.64-7.70 \mathrm{~m}(3 \mathrm{H}, \mathrm{Ar}-\mathrm{H}), 7.74-7.81 \mathrm{~m}(2 \mathrm{H}, \mathrm{Ar}-\mathrm{H}), 7.90-7.96 \mathrm{~m}(2 \mathrm{H}, \mathrm{Ar}-\mathrm{H}), 8.04 \mathrm{~d}$ $(1 \mathrm{H}, \mathrm{Ar}-\mathrm{H}, J=16.8 \mathrm{~Hz}), 8.26 \mathrm{~d}(2 \mathrm{H}, \mathrm{Ar}-\mathrm{H}, J=8.2 \mathrm{~Hz}) .{ }^{13} \mathrm{C}$ NMR spectrum, $\delta_{\mathrm{C}}, \mathrm{ppm}: 112.7,115.5,119.2$, 
RASĀYAN J. Chem.

Vol. 13 | No. 1 |601 - 609| January - March | 2020

$120.2,120.2,120.4,123.1,124.3,125.0,125.3,126.0,126.1,126.4,127.0,127.4,127.7,128.4,128.8$, 129.5, 129.9, 130.1, 131.3, 138.8, 139.5, 145.5, 151.2, 154.9, 171.7, 185.6. Mass spectrum, $\mathrm{m} / \mathrm{z}$ (Irel, \%): $519[\mathrm{M}+\mathrm{H}]$; Found, \%: C 78.75; H 4.28; N 10.80. $\mathrm{C}_{34} \mathrm{H}_{22} \mathrm{~N}_{4} \mathrm{O}_{2}$. Calculated, \%: C 78.79; H 4.24; N 10.87.

\section{Biological Activity}

All the synthesized compounds $\mathbf{5 a - 5 h}$ and $\mathbf{7} \mathbf{a}-\mathbf{7} \mathbf{j}$ were screened in-vitro for their antibacterial activity, at $20 \mu \mathrm{g} / \mathrm{mL}$, and $40 \mu \mathrm{g} / \mathrm{mL}$ concentrations. The Zone of inhibition (in $\mathrm{mm}$ ) was compared with standard drug Ciprofloxacin. Among all, the compounds $5 \mathbf{b}, \mathbf{5 c}, \mathbf{5 e}, \mathbf{7 b}, \mathbf{7 c}, \mathbf{7 d}$, and $\mathbf{7 e}$ were exhibited better activity against all bacterial organisms and compounds $5 \mathbf{b}, \mathbf{5 c}, \mathbf{5 e}, \mathbf{7 b}, \mathbf{7 c}, \mathbf{7 d}$, and $7 \mathrm{e}$ were shown maximum zone of inhibition. The compounds as $\mathbf{5 a}, \mathbf{5 d}, \mathbf{5 f}, \mathbf{7 f}, \mathbf{7 h}$, and $\mathbf{7 i}$ shown a moderate zone of inhibition, remaining compounds have shown poor activity against bacterial strains.

Furthermore, the antifungal activity of synthesized compounds $\mathbf{5 a - 5 h}$ and $\mathbf{7 a - 7 \mathbf { j }}$ were tested against three pathogenic fungi at $50 \mu \mathrm{g} / \mathrm{mL}$ concentrations. The zone of inhibition compares to standard drug Amphotericin-B. Among all, the compounds $\mathbf{5 b}, \mathbf{7 b}$ and $\mathbf{7} \mathbf{c}$ with flouro substitution on ring showed maximum activity. Compounds $\mathbf{7} \mathbf{h}, \mathbf{7} \mathbf{i}$ with carbonyl substitution on a ring was active against $A$. flavus

Table-4: Antimicrobial Activity of Synthesized Compounds $\mathbf{5 a - 5 h}$ and $\mathbf{7 a - 7} \mathbf{j}$

\begin{tabular}{|c|c|c|c|c|c|c|c|c|c|c|c|}
\hline & \multicolumn{11}{|c|}{ Zone of Inhibiton $(\mathrm{mm})$, Concentration $(\mu \mathrm{g} / \mathrm{mL})$} \\
\hline & \multicolumn{4}{|c|}{ Gram-positive Bacteria } & \multicolumn{4}{|c|}{ Gram-negative Bacteria } & \multicolumn{3}{|c|}{ Fungi } \\
\hline & \multicolumn{2}{|c|}{ S. aeures } & \multicolumn{2}{|c|}{ B. subtilis } & \multicolumn{2}{|c|}{ K. pneumoniae } & \multicolumn{2}{|c|}{ E. coli } & \multirow{2}{*}{$\begin{array}{c}A . \\
\text { nigerzeae } \\
50\end{array}$} & \multirow{2}{*}{$\begin{array}{c}\begin{array}{c}A . \\
\text { flavus }\end{array} \\
50\end{array}$} & \multirow{2}{*}{$\begin{array}{c}F . \\
\text { oxysporum } \\
50\end{array}$} \\
\hline Compound & 20 & 40 & 20 & 40 & 20 & 40 & 20 & 40 & & & \\
\hline 5a & 15 & 28 & 12 & 24 & 18 & 28 & 10 & 25 & 10 & 9 & 12 \\
\hline $\mathbf{5 b}$ & 14 & 27 & 15 & 28 & 22 & 34 & 15 & 35 & 13 & 11 & 13.7 \\
\hline $5 \mathrm{c}$ & 13 & 20 & 14 & 21 & 21 & 30 & 13 & 31 & 9 & 9.5 & 10 \\
\hline 5d & 11 & 20 & 12 & 25 & 20 & 29 & 11 & 27 & 7 & 8 & 12 \\
\hline $5 e$ & 13 & 21 & 13 & 24 & 23 & 31 & 12 & 30 & 8 & 7.4 & 11 \\
\hline $5 f$ & 11 & 19 & 11 & 21 & 14 & 30 & 14 & 28 & 7.5 & 9.2 & 10.4 \\
\hline $5 \mathrm{~g}$ & 9 & 20 & 9 & 20 & 11 & 22 & 9 & 21 & 8 & 9 & 10 \\
\hline $5 \mathrm{~h}$ & 10 & 21 & 10 & 21 & 14 & 21 & 11 & 17 & 11 & 10 & 12 \\
\hline $7 a$ & 9 & 22 & 7 & 20 & 12 & 24 & 8 & 22 & 7 & 9 & 11 \\
\hline $7 b$ & 13 & 26 & 15 & 26 & 19 & 28 & 14 & 29 & 13 & 11.7 & 14 \\
\hline $7 \mathrm{c}$ & 14 & 25 & 14 & 25 & 17 & 25 & 15 & 30 & 13 & 11.2 & 13.4 \\
\hline $7 d$ & 12 & 24 & 14 & 23 & 16 & 27 & 15 & 28 & 11 & 10.4 & 12 \\
\hline $7 e$ & 14 & 26 & 14 & 24 & 18 & 26 & 14 & 29 & 8 & 9 & 10 \\
\hline $7 f$ & 11 & 21 & 11 & 19 & 14 & 21 & 15 & 25 & 11 & 9.8 & 9.4 \\
\hline $7 \mathrm{~g}$ & 9 & 20 & 10 & 20 & 13 & 20 & 12 & 21 & 8 & 9 & 10 \\
\hline $7 \mathrm{~h}$ & 10 & 21 & 12 & 21 & 19 & 28 & 14 & 24 & 10 & 14 & 11.4 \\
\hline $7 i$ & 11 & 22 & 13 & 20 & 18 & 26 & 12 & 27 & 11.2 & 13.7 & 12.4 \\
\hline $7 \mathbf{j}$ & 8 & 21 & 13 & 19 & 18 & 19 & 14 & 23 & 7.5 & 8.2 & 11 \\
\hline Ciproflaxacin & 15 & 28 & 16 & 30 & 23 & 35 & 18 & 35 & - & - & - \\
\hline $\begin{array}{c}\text { Amphotericin- } \\
B \\
\end{array}$ & - & - & - & - & - & - & - & - & 16.3 & 15.6 & 16.2 \\
\hline
\end{tabular}

\section{Biological Assay}

\section{Antibacterial Activity}

The synthesized scaffolds $\mathbf{5 a - 5 h}$ and $\mathbf{7 a - 7 j}$ were screened for their antibacterial activity against bacterial strains such as Pseudomonas aeruginosa, Escherichia coli, Bacillus subtilis and Staphylococcus aeureus at a concentration of $20 \mu \mathrm{g} / \mathrm{mL}$ and $40 \mu \mathrm{g} / \mathrm{mL}$. The cultures were diluted with $4 \%$ autoclaved saline and the final volume was made with concentration approximately $10^{5}-10^{6} \mathrm{CFU} / \mathrm{mL}$. The synthesized compounds were diluted in DMSO. According to the procedures described agar disc diffusion method ${ }^{19}$. The synthesized compounds were added to the disc and plates were incubated at $37^{\circ} \mathrm{C}$ for $24 \mathrm{hr}$. Zones of inhibition were measured in $(\mathrm{mm})$. All experiments were carried out in triplicates. 
RASĀYAN J. Chem.

Vol. 13 | No. 1 |601 - 609| January - March | 2020

\section{Antifungal Activity}

The antifungal activity of synthesized compounds $\mathbf{5 a - 5 h}$ and $\mathbf{7 a - 7} \mathbf{j}$ was tested against three pathogenic fungi such as Fusarium oxysporum, Aspergillus niger, and Penicillium italicum, by the poison plate technique at a concentration of $50 \mu \mathrm{g} / \mathrm{mL}$. Three kinds of fungi were incubated in PDA at $25 \pm 1{ }^{\circ} \mathrm{C}$ for 5 days to get new mycelium for antifungal assay, then a mycelia as disks of approximately $0.45 \mathrm{~cm}$ diameter cut from the culture medium were picked up with a sterilized inoculation needle and inoculated in the center of PDA plate. The test compounds were dissolved in DMSO $(10 \mathrm{~mL})$ then added to the Potato Dextrose Agar medium (PDA, $20 \mathrm{~mL}$ ). The final concentration of compounds in the medium was adjusted to $50 \mu \mathrm{g} / \mathrm{mL}$. The inoculated plates were incubated at $25 \pm 1{ }^{\circ} \mathrm{C}$ for 5 days. DMSO was diluted with sterilized distilled water and used as a control, while Amphotericin-B. $(50 \mu \mathrm{g} / \mathrm{mL})$ was used as a standard control for each treatment three replicates of experiments were carried out. The radial growth of the fungal colonies was measured on the sixth day.

\section{CONCLUSION}

In conclusion, we herein the report the new scaffolds $\mathbf{5 a - 5 h}$ and $\mathbf{7 a - 7} \mathbf{j}$ were synthesized by using a microwave irradiation method to obtained better yield as compared to conventional heating. The reaction carried out under green conditions. They evaluated their antimicrobial activity and the compounds $\mathbf{5 b}, \mathbf{5} \mathbf{c}$, $\mathbf{5 e}, \mathbf{7 b}, \mathbf{7 c}, \mathbf{7 d}$, and $\mathbf{7 e}$ were found to be good antibacterial activity and compounds $5 \mathbf{b}, \mathbf{7 b}$ and $7 \mathbf{c}$ were exhibited good antifungal activity. The $\mathbf{7 b}$ and $\mathbf{7} \mathbf{c}$ were exhibited better antimicrobial activity.

\section{ACKNOWLEDGMENT}

We are thankful to the Head, Department of Chemistry, Osmania University, for providing laboratory facilities and CFRD OU, for providing spectral analysis. We are thankful to the University Grants Commission, New Delhi, for their financial support and one of the authors, Ravinder Dharavath is thankful to CSIR-New Delhi, India, for financial support. D. Ashok is thankful to UGC, New Delhi for the award of UGC-BSR fellowship.

\section{REFERENCES}

1. V.A. Ostrovskii, G.I. Koldobskii, R.E. Trifonov, Comprehensive Heterocyclic Chemistry, 6, 257(2008), DOI:10.1016/B978-008044992-0.00517-4

2. D. Ashok, N. Nagaraju, M. Sarasija, B. Vijaya Lakshmi, Journal of the Mexican Chemical Society, 63(4), 123(2019), DOI: $10.29356 /$ jmcs.v63i4.448

3. D. Ashok, N. Nagaraju, M. Sarasija, B. Vijaya Lakshmi, Journal of the Serbian Chemical Society, 83(12), 1305(2018), DOI:10.2298/JSC170825064A

4. D. Ashok, N. Nagaraju, B. Vijaya Lakshmi, M. Sarasija, Russian Journal of General Chemistry, 89, 1905 (2019), DOI: 10.1134/S1070363219090275

5. P.B. Mohite, V.H. Bhaskar, Advanced Pharmaceutical Bulletin, 2, 31(2012), DOI: $10.5681 \% 2 \mathrm{Fapb} .2012 .005$

6. A. Rajasekaran, S. Murugesan, K. Ananda Rajagopal, Arch. Pharmacal Res., 29, 535(2006), DOI:10.1007/BF02969261

7. J. Adamec, K. Waisser, J. Kunes, J. Kaustova, Archiv der Pharmazie: An International Journal Pharmaceutical and Medicinal Chemistry, 338(8), 385(2005), DOI:10.1002/ardp.200400967

8. R.S. Upadhayaya, S. Jain, N. Sinha, N. Kishore, R. Chandra, S.K. Arora, European Journal of Medicinal Chemistry, 39(7), 579(2004), DOI:10.1016/j.ejmech.2004.03.004

9. R. Romagnoli, P.G. Baraldi, M.K. Salvador, D. Preti, M. Aghazadeh Tabrizi, A. Brancale, X.H. Fu, J. Li, S.Z. Zhang, E. Hamel, R. Bortolozzi, Journal of Medicinal Chemistry, 55(1), 475(2012), DOI: $10.1021 / \mathrm{jm} 2013979$

10. B. Le Bourdonnec, C. Cauvin, E. Meulon, S. Yous, J. F. Goossens, F. Durant, J. P. Hénichart, Journal of Medicinal Chemistry, 45(21), 4794(2002), DOI:10.1021/jm010457z

11. G. Bringmann, A.J. Price Mortimer, P.A. Keller, M.J. Gresser, J. Garner, M. Breuning, Angewandte Chemie International Edition, 44(34), 5384(2005), DOI:10.1002/anie.200462661

12. N.E. Leadbeater, M. Marco, Organic Letters, 4(17), 2973(2002), DOI:10.1021/o10263907 
RASĀYAN J. Chem.

Vol. 13 | No. 1 |601 - 609| January - March | 2020

13. L. Liu, Y. Zhang, Y. Wang, The Journal of Organic Chemistry, 70(15), 6122(2005), DOI: $10.1021 /$ jo050724z

14. M. Mondal, U. Bora, Green Chemistry, 14(7), 1873(2012), DOI:10.1039/C2GC35401B

15. U. Schoen, J. Messinger, S. Eichner, A. Kirschning, Tetrahedron Letters., 49(20), 3204(2008), DOI: 10.1016/j.tetlet.2008.03.094

16. C.B. Patneedi, K. Durga Prasadu, R.S.K. Sharma, D. Chandra Sekhar, D.V. Venkata Rao, Rasayan Journal of Chemistry, 8, 176(2015).

17. O.S. Chavan, S.A. Jadhav, M.G. Shioorkar, S.B. Chavan, M.A. Baseer, D.B. Shinde, Rasayan Journal of Chemistry, 8(2), 194(2015).

18. D. Ashok, E.V.L. Madhuri, M. Sarasija, S.S. Kanth, M. Vijjulatha, M.D. Alaparthi, S.R. Sagurthi, RSC Advances., 7(41), 25710(2017), DOI:10.1039/c7ra01550j

19. K. Hassan, M. El Bagoury, Rasayan Journal of Chemistry, 11(1), 238(2018), DOI: 10.7324/RJC.2018.1112019

[RJC-5490/2019] 